\title{
I cento fascicoli delle „Quellen und Forschungen aus italienischen Archiven und Bibliotheken“, 1898-2020
}

\section{Riflessioni di una medievista}

\begin{abstract}
On the occasion of the 100th issue of the „Quellen und Forschungen aus italienischen Archiven und Bibliotheken“, the article offers a retrospective look at the journal, with a focus on the medieval section. It highlights the presentation or edition of Vatican sources, both as a reflection of the Institute's research and as a constant distinctive feature in the context of international journals. At the same time, the author stresses the journal's openness to new topics following trends in medieval studies, alongside well-established issues, and the increasingly intense internationalisation resulting from collaboration with other researchers and institutes based in Rome, Italy and abroad. Finally, it expresses the hope that the journal may continue to be a means of fostering research on primary sources, especially in the context of the Repertorium Germanicum.
\end{abstract}

A poca distanza dalla celebrazione nel 2013 dei 125 anni dell'Istituto Storico Germanico di Roma (DHI), ${ }^{1}$ si festeggia nel 2020 il centesimo fascicolo del suo organo, le „Quellen und Forschungen aus italienischen Archiven und Bibliotheken“ (QFIAB). Per tale occasione proverò a svolgere alcune riflessioni sul significato della rivista nella medievistica, in particolare nel quadro delle relazioni scientifiche italo-tedesche; le mie valutazioni, fondate come sono sulle pubblicazioni e non sulle fonti d'archivio del DHI, risulteranno un po' impressionistiche, specie per chi da anni e con competenza lavora nell'Istituto, le porgo quindi come un piccolo contributo alla ricorrenza, da osservatrice esterna e lettrice affezionata.

I cento numeri coprono gli anni dal 1898 al 2020, con una pausa decennale alla fine delle due guerre mondiali, rispettivamente nel 1915-1923 e nel 1945-1953. Inaugurate come bollettino discreto, senza squilli di tromba o documenti programmatici, „per far conoscere l'attività secondaria dei membri dell'Istituto“,2 di quest'ultimo le

1 Jahresbericht 2013, in: QFIAB 14 (2014), pp. VII sg. Nel 2013 si sono contemporaneamente celebrati i 60 anni dalla riapertura dell'Istituto (1953), dopo la chiusura decennale determinata dalla guerra. 2 Reinhard Elze, I cento anni dell'Istituto Storico Germanico in Roma, in: Deutsches Historisches Institut Rom, Istituto Storico Germanico, 1888-1988, Roma 1988, pp. 59-94, a p. 64.

Kontakt: Daniela Rando, daniela.rando@unipv.it 
QFIAB rispecchiano la vita, al punto che riferirne puntualmente significherebbe fare la storia del DHI medesimo, peraltro più volte ripercorsa. ${ }^{3}$ L'Istituto balza in primo piano dagli „Jahresberichte“, pubblicati a partire dal fascicolo 7 (1904), ${ }^{4}$ ma in parte prefigurati nelle „Nachrichten“ dei volumi immediatamente precedenti. ${ }^{5} \mathrm{Fu}$ Paul F. Kehr, assunta nel 1903 la guida dell'Istituto, ad introdurre lo „Jahresbericht“, dandogli una struttura destinata a lunga fortuna: indicazione degli organi di governo e di controllo, collaboratori, pubblicazioni, biblioteca, ricerche svolte per studiosi esterni, ricerche di studiosi interni, iniziative, ringraziamenti a istituzioni ed enti tedeschi e italiani. Dalle sette pagine di Kehr gli „Jahresberichte“ sono via via lievitati fino alle settanta pagine degli ultimi fascicoli, corredate di paragrafi e indice tematico: ${ }^{6}$ specchio di un istituto che ha visto crescere i suoi compiti e il suo personale - certo non in proporzione!, le sue relazioni e il suo irraggiamento internazionali.

Si tratta di resoconti scritti con un occhio rivolto ai burocrati ministeriali, ma di per sé tutt'altro che burocratici. Vi si disegna l'esistenza concreta dell'Istituto - i problemi finanziari, gli anni tra le due guerre e la ricerca di una sede, fino alle discussioni relative alla proposta di trasformazione in fondazione (Stiftung) ${ }^{-7}$ senza però negarsi qualche guizzo di attualità; ad esempio quando si accenna ai ritardi provocati dalle stamperie romane nel 1911-1912, ${ }^{8}$ alle „Bestrebungen und Wirren der Hochschulund der Sozialpolitik“" nel ' $68^{9}$ o alle tensioni politiche che attraversarono gli ,anni di piombo', con uno stato di allarme determinato nel 1977 dalla fuga di Herbert Kappler e dagli avvenimenti seguiti al rapimento e assassinio di Hanns Martin Schleyer. ${ }^{10}$

In riferimento specifico all'organizzazione delle ricerche, negli „Jahresberichte“ del nuovo millennio fa capolino la parolina Drittmittel (finanziamenti da parte di terzi), insieme con la digitalizzazione e il discorso di genere - dal 2006 s'è iniziato a riportare la percentuale del rapporto tra uomini e donne impiegati al DHI. ${ }^{11}$ Professionali e politicamente corretti, gli „Jahresberichte“ conservano un tocco umano: fa sorridere la notizia fornita da Reinhard Elze nel non lontano 1980 sui temporali che il 23 e 31 dicembre 1979 avevano sradicato tre alberi del parco - un eucalipto, un pino e

3 Gerd Tellenbach, Zur Geschichte des preußischen historischen Instituts in Rom (1888-1936), in: QFIAB 50 (1971), pp. 382-419, ora in: id., Ausgewählte Abhandlungen und Aufsätze, vol. 1, Stuttgart 1988, pp. 182-218; Elze, I cento anni (vedi nota 2).

4 Jahresbericht des Historischen Instituts, in: QFIAB 7 (1904), pp. 1-7.

5 Cfr. Nachrichten, in: QFIAB 1 (1898), pp. $154 \mathrm{sg}$.

6 Jahresbericht 2015, in: QFIAB 96 (2016), pp. IX-LXX; Jahresbericht 2016, in: QFIAB 97 (2017), pp. IX-LXVIII.

7 Cfr. Elze, I cento anni (vedi nota 2), pp. 72-79.

8 Jahresbericht des Historischen Instituts 1911-12, in: QFIAB 15 (1913), pp. V sg.

9 Jahresbericht 1968, in: QFIAB 49 (1969), pp. VII-XV, a p. XIII: A proposito di Wolfgang Schieder ad Heidelberg, il direttore Tellenbach scriveva: „Er ist freilich, obgleich er offiziell nicht zur Universität Heidelberg gehört, nicht unberührt geblieben von den Bestrebungen und Wirren der Hochschul- und der Sozialpolitik.“.

10 Jahresbericht 1977, in: QFIAB 58 (1978), pp. VII-XXIII, a p. VIII.

11 Lo si riferisce nello Jahresbericht 2005, in: QFIAB 86 (2006), pp. IX-LV, a p. XV. 
una mimosa. ${ }^{12}$ Notizia dal sapore antico - forse un calco arguto dagli annali monastici altomedievali, ma pervasa nel contempo di quella sensibilità per la natura che gli italiani invidiano ai tedeschi. La lettura degli „Jahresberichte“ è insomma illuminante perché, scontate le convenzioni del genere, si apre alle condizioni reali del fare storia, immettendo il lettore nel sostrato che nutre e sorregge la rivista, nel contesto vivo della produzione di fascicoli e singoli saggi.

Riguardo al Medioevo, se i primi numeri sono sostanzialmente dedicati alla presentazione di fonti vaticane, i volumi successivi si arricchiscono di temi molteplici. L'edizione di fonti (Quellen), il primo dei due corni del titolo originario rimasto finora immutato, è legata ad origine e scopi della primitiva stazione prussiana a Roma dopo l'apertura degli archivi vaticani e occupa uno spazio consistente soprattutto nei primi decenni. Nel 1925 Helene Wieruszowski approntò un lungo elenco delle fonti pubblicate nei primi 17 numeri $^{13}$ e altrettanto si fece negli indici successivi del 1960 e del 1973. Delle 130 pagine a stampa di quest'ultimo, che ingloba i precedenti e censisce documenti editi dal 772 al 1914, ben cento sono le pagine occupate dall'età medievale. ${ }^{14}$ Tali fonti sono state solo in parte ristampate all'interno di raccolte più ampie come i „Diplomata“ di Federico I, ${ }^{15}$ sicché rimangono tuttora preziose; insieme con l'eccezionale lavoro sedimentato nel „Repertorium Germanicum“ e nel „Repertorium Germanicum Poenitentiariae“ (oltreché nei „Nuntiaturberichte“) testimoniano il tenace impegno del DHI nella ricerca di base. Il peso delle edizioni e delle notizie di documenti è gradualmente diminuito, anche se non mancano regesti o edizioni nemmeno negli ultimi numeri, specie in appendice a singoli saggi; ma il baricentro si è decisamente spostato, segno dei nuovi orientamenti dell'Istituto nei confronti della ricerca di base e delle nuove tecniche di edizione digitale.

Le ricerche (Forschungen) evocate dal secondo corno del titolo si sono mosse nei decenni lungo le linee via via più innovative della medievistica. Un nucleo solido e costante di saggi ruota attorno alle fonti vaticane e quindi alla storia del papato e della curia (procuratori, abbreviatori, cardinali etc.), al „Repertorium Germanicum“ e agli strumenti di accesso agli archivi vaticani come lo schedario Garampi, all',Italia Pontificia“. ${ }^{16}$ Accanto ad esso, ampio spazio hanno avuto le ricerche sulla

12 „Die schweren Stürme am Jahresende (23. und 31. 12.) haben im Institutsgarten drei große Bäume entwurzelt (Eukalyptus, Pinie, Mimose), an den Gebäuden jedoch glücklicherweise nur geringen Schaden angerichtet“: Jahresbericht 1979, in: QFIAB 60 (1980), pp. VII-XXIV, a p. IX.

13 Helene Wieruszowski, Chronologisches Verzeichnis der in den Bänden 1-17 der „Quellen und Forschungen“ abgedruckten Urkunden und Aktenstücke, in: QFIAB 17 (1914-1924), pp. 283-342, alle pp. 290-324 (il mio calcolo riguarda gli anni 772-1500).

14 Register zu den Bänden 1-50: 1898-1971, bearb. von Brigitte Szabó-Bechstein, Tübingen 1973, pp. 1-101 (fino al 1500).

15 Marlene Polock, Unbekannte Kaiserdiplome für Montefiascone, in: QFIAB 65 (1985), pp. 105-132, alle pp. 126-129 (Anhang I), riedito in: MGH DD F I., nr. 915.

16 A titolo d'esempio Walther Holtzmann, Kanonistische Ergänzungen zur Italia pontificia I-IV, in: QFIAB 37 (1957), pp. 55-102; Walther Holtzmann, Kanonistische Ergänzungen zur Italia pon- 
Toscana - uno dei campi d'indagine promossi da Kehr nel 1904-1905 ${ }^{17}$ - come pure la storia imperiale, le Reichssachen, come si usava dire nel primo Novecento. ${ }^{18}$ Negli anni Settanta si coglie un accento nuovo posto sulla „storia sociale“, affiorante fin dal titolo sia in Arnold Esch, che nel 1973 sfruttava in tale direzione le fonti agiografiche, ${ }^{19}$ contemporaneamente agli studi di André Vauchez sulla santità nel Medioevo, sia in Norbert Kamp. ${ }^{20}$ Emergono poi i testamenti come fonte per la storia sociale e religiosa (1990), ${ }^{21}$ un indirizzo anch'esso presente nella storiografia francese (Chiffoleau) e italiana (Bartoli Langeli). ${ }^{22}$ Alla svolta del millennio si affermano le fonti iconografiche nel pregevole saggio del 2001 di Patrizia Carmassi, ${ }^{23}$ nel saggio di Uwe Israel del $2004^{24}$ e in quello di Stefan Bauer sul rapporto testo-monumento nel 2011. ${ }^{25} \mathrm{Vi}$ si affiancano i rapporti interculturali, con Daniel König (2010), Anna Esposito (2011), Arnold Esch (2012), ${ }^{26}$ i rituali e la comunicazione simbolica (Christoph Dartmann

tificia V-X, in: QFIAB 38 (1958), pp. 67-175; Hermann Diener, Schedario Garampi. Eine Exzerptensammlung des 18. Jahrhunderts als Hilfsmittel zur Erschließung des Vatikanischen Archivs, in: QFIAB 62 (1982), pp. 204-221.

17 Arnold Esch, Forschungen in der Toskana, in: Reinhard Elze/Arnold Esch (a cura di), Das Deutsche Historische Institut in Rom 1888-1988, Tübingen 1990 (Bibliothek des Deutschen Historischen Instituts in Rom 70), pp. 191-210.

18 Elze, I cento anni (vedi nota 2), p. 74, circa le Kaiserurkunden und Reichssachen raccolte fino agli anni Sessanta.

19 Arnold Esch, Die Zeugenaussagen im Heiligsprechungsverfahren für S. Francesca Romana als Quelle zur Sozialgeschichte Roms im frühen Quattrocento, in: QFIAB 53 (1973), pp. 93-151.

20 Norbert Kamp, Die sizilischen Verwaltungsreformen Kaiser Friedrichs II. als Problem der Sozialgeschichte, in: QFIAB 62 (1982), pp. 119-142.

21 Martin Bertram, Mittelalterliche Testamente. Zur Entdeckung einer Quellengattung in Italien, in: QFIAB 68 (1988), pp. 509-545; id., Hundert Bologneser Testamente aus einer Novemberwoche des Jahres 1265, in: QFIAB 69 (1989), pp. 80-110; id., Bologneser Testamente. Erster Teil: Die urkundliche Überlieferung, in: QFIAB 70 (1990), pp. 151-233; id., Bologneser Testamente. Zweiter Teil: Sondierungen in den Libri memoriali, in: QFIAB 71 (1991), pp. 195-240.

22 Attilio Bartoli Langeli (a cura di), Nolens intestatus decedere: il testamento come fonte della storia religiosa e sociale. Atti dell'incontro di studio (Perugia, 3 maggio 1983), Perugia 1985; Jacques Chiffoleau, La comptabilité de l'Au-Delà. Les hommes, la mort et la religion dans la région d'Avignon à la fin du Moyen Âge (vers 1320 - vers 1480), Roma 1980.

23 Patrizia Carmassi, Die hochmittelalterlichen Fresken der Unterkirche von San Clemente in Rom als programmatische Selbstdarstellung des Reformpapsttums. Neue Einsichten zur Bestimmung des Entstehungskontexts, in: QFIAB 81 (2001), pp. 1-66.

24 Uwe Israel, Der Papst und die Urkunde an der Wand. Innozenz III. (1198-1216) in Subiaco, in: QFIAB 84 (2004), pp. 69-102.

25 Stefan Bauer, Quod adhuc extat. Le relazioni tra testo e monumento nella biografia papale del Rinascimento, in: QFIAB 91 (2011), pp. 217-248.

26 Daniel G. König, Zur Ausstrahlung des Papsttums in die mittelalterliche arabisch-islamische Welt. Eine Evaluation der arabisch-islamischen Berichterstattung zum Bischof von Rom, in: QFIAB 90 (2010), pp. 1-52; Anna Esposito, Gli ebrei aschenaziti a Roma nel primo Rinascimento, in: QFIAB 91 (2011), pp. 249-276; Arnold Esch, Der Handel zwischen Christen und Muslimen im MittelmeerRaum. Verstöße gegen das päpstliche Embargo geschildert in den Gesuchen an die Apostolische Pönitentiarie (1439-1483), in: QFIAB 92 (2012), pp. 85-140. 
2006). ${ }^{27}$ Con Lucas Burkart e Thomas Ertl l'attenzione si sposta sulla cultura materiale (2006-2007), ${ }^{28}$ fino alla storia globale (Michael Borgolte 2013) ${ }^{29}$ e dell'ambiente (Martin Bauch 2015). ${ }^{30}$ Né mancano le nuove forme di comunicazione - blog e social media. ${ }^{31}$ Particolare cura è riservata all'umanesimo grazie ai contributi sia del mitico „bibliotecario scientifico“ Hermann M. Goldbrunner, dagli stretti contatti con Paul O. Kristeller e promotore dell'incipitario Bertalot, ${ }^{32}$ sia di Mariarosa Cortesi e di altri; il progetto dei „Libri legales“ iniziato da Manlio Bellomo ${ }^{33}$ e la collaborazione dal 1979 al „Vatican Project“, cioè alla catalogazione dei manoscritti canonistici della Biblioteca Vaticana iniziata da Stephan Kuttner, ${ }^{34}$ mostrano la varietà di temi e l'ampiezza d'orizzonti delle QFIAB. Scorrendo i suoi indici è dunque possibile seguire le traiettorie della medievistica internazionale nel corso del Novecento e di questo primo scorcio di secolo, a testimonianza, mi pare, della capacità della rivista - quindi del DHI - di partecipare attivamente al dibattito scientifico. Nonostante i timori e le riserve di Elze nel 1988 circa un qualche ritardo, ${ }^{35}$ le QFIAB sono anzi divenute in alcuni casi antesignane della ricerca su nuove tematiche, grazie alla freschezza di idee apportate dai giovani borsisti che ad esse hanno cooperato. È questa la risorsa che i direttori del DHI - da Gerd Tellenbach a Reinhard Elze, da Arnold Esch, Michael Matheus a Martin Baumeister - hanno voluto e saputo valorizzare, facendone un punto di forza specifico delle giovani e sempre ringiovanite QFIAB. La formazione di studiosi al lavoro scientifico, che Kehr nel 1907 considerava fra le ragioni principali dell'esistenza dell'Istituto, ${ }^{36}$ si riverbera nella fisionomia delle QFIAB rispetto ad altre riviste, tanto nei

27 Christoph Dartmann, Adventus ohne Stadtherr. ,Herrschereinzüge“ in den italienischen Stadtkommunen, in: QFIAB 86 (2006), pp. 64-94.

28 Lucas Burkart, Das Verzeichnis als Schatz. Überlegungen zu einem Inventarium Thesauri Romane Ecclesie der Biblioteca Apostolica Vaticana (Cod. Ottob. lat. 2516, fol. 126r-132r), in: QFIAB 86 (2006), pp. 144-207; Thomas Ertl, Stoffspektakel. Zur Funktion von Kleidern und Textilien am spätmittelalterlichen Papsthof, in: QFIAB 87 (2007), pp. 139-185.

29 Michael Borgolte, Carlo Magno e la sua collocazione nella storia globale, in: QFIAB 93 (2013), pp. 1-26.

30 Martin Bauch, Der Regen, das Korn und das Salz: die Madonna di San Luca und das Wettermirakel von 1433. Eine klimahistorische Fallstudie zu Bologna und Italien in den 1430er Jahren, in: QFIAB 95 (2015), pp. 183-212.

31 Martin Bauch, Nuove forme di comunicazione per medievisti: Blog scientifici e social media. Annotazioni su un workshop svoltosi presso il DHI Roma, in: QFIAB 94 (2014), pp. 380-384.

32 L'incipitario Bertalot è stato pubblicato con il titolo di „Initia Humanistica Latina“. Sul suo promotore si veda il „Nachruf“ di Arnold Esch, Hermann Goldbrunner, 1933-2004, in: QFIAB 84 (2004), pp. XLIII-XLVIII.

33 Jahresbericht 1986, in: QFIAB 67 (1987), pp. VII-XXIII, a p. XIV.

34 Kuttner lavorava allora all'Institute of Medieval Canon Law di Berkeley. Sull'iniziativa cfr. Jahresbericht 1980, in: QFIAB 61 (1981), pp. VII-XXVI, a p. IX.

35 Elze, I cento anni (vedi nota 2), p. 94.

36 Denkschrift über die Zukunft des Historischen Instituts in Rom (verfasst von Paul F. Kehr, April 1907), in: Hubert Houben, Hundert Jahre deutsche Kastellforschung in Süditalien, in: QFIAB 84 (2004), pp. 103-136, alle pp. 131-135, in particolare pp. 134 sg.); Elze, I cento anni (vedi nota 2), p. 81. 
diversi saggi quanto nei progetti di collaboratori e borsisti, illustrati negli „Jahresberichte“ e ora pure più ampiamente nel „Forum“.

La rivista ha avuto ben presto vocazione internazionale, accogliendo francesi (Deprez 1900, Ménager 1959), ${ }^{37}$ inglesi (Luttrell 1976), ${ }^{38}$ americani (Brentano 1961, Trexler 1979, 1985) ${ }^{39}$ e molti italiani. Questi ultimi, presenti con Andrea Da Mosto fin dal $1903,{ }^{40}$ sono diventati sempre più numerosi dopo la ripresa del 1953, tanto che, per fare solo qualche esempio, nelle QFIAB del 1993 figurano cinque autrici e autori italiani, ${ }^{41}$ nel 2012 ben sette su quindici. ${ }^{42}$ Tale frequenza è conseguenza diretta delle borse di studio assicurate dalla fine degli anni Sessanta a giovani ricercatori italiani, i cui studi sono confluiti quasi naturalmente nella rivista. Ne è derivato un fertile dialogo scientifico a partire da Vito Fumagalli, il primo assistente italiano dell'Istituto, che vi lavorò dal '66 al '69.43 I saggi di Fumagalli vennero accolti nelle QFIAB e la sua abilitazione su „Le origini di una grande dinastia feudale. Adalberto-Atto di Canossa“ fu addirittura pubblicata in italiano nella collana del DHI. Il volume doveva molto all'Adels- e Personenforschung del Freiburger Arbeitskreis e al suo capofila, Gerd Tellenbach, allora energico e creativo direttore del DHI, il quale aveva attirato a Roma diversi suoi allievi, tutti poi autori di articoli destinati alle QFIAB: Wilhelm Kurze, Hermann Diener e Karl Schmid, quest'ultimo già Gastdozent al DHI nei due anni precedenti l'arrivo di Fumagalli. Non meraviglia quindi che quest'ultimo dedicasse la citata abilitazione, oltre che a Ottorino Bertolini e Cinzio Violante, appunto a Tellenbach. ${ }^{44}$

37 Eugène Déprez, Recueil des documents pontificaux conservés dans diverses archives d'Italie (XIII ${ }^{\mathrm{e}}$ et XIVe siècles), in: QFIAB 3 (1900), pp. 103-128, 255-307. Seguirono Léon-Robert Ménager, Les fondations monastiques de Robert Guiscard, duc de Pouille et de Calabre, in: QFIAB 39 (1959), pp. 1-116, André Guillou, Zwei Katepansurkunden aus Tricarico, in: QFIAB 41 (1961), pp. 1-28 e diversi altri. 38 Anthony Luttrell, Frederick II and Paolino de Malta: 1235, in: QFIAB 55/56 (1976), pp. 405-409. 39 Robert Brentan o, Peter of Assisi as Witness, in: QFIAB 41 (1961), pp. 323-325; id., A New Roman Senator, in: QFIAB 52 (1972), pp. 789-796; Richard C. Trexler, The Episcopal Constitutions of Antoninus of Florence, in: QFIAB 59 (1979), pp. 244-272; id., Herald of the Ciompi. The Authorship of an Anonymous Florentine chronicle, in: QFIAB 65 (1985), pp. 159-191.

40 Andrea Da Mosto, Ordinamenti delle soldatesche dello Stato Romano dal 1430 al 1470, in: QFIAB 5 (1903), pp. 19-34; id., Ordinamenti militari delle soldatesche dello stato romano nel secolo XVI, in: QFIAB 6 (1904), pp. 72-133. Lo seguì nel 1907 Augusto Gaudenzi, Un nuovo manoscritto delle collezioni irlandese e pseudoisidoriana e degli estratti bobbiesi, in: QFIAB 10 (1907), pp. 370-379.

41 Si tratta di Ivana Ait, Maria Pia Alberzoni, Aldo Messina, Francesco Mottola e Filippo Tamburini, presenti in: QFIAB 73 (1993).

42 Sono i medievisti Eugenio Riversi e Pierpaolo Bonacini, insieme con Roberto Zapperi, Patrizio Foresta, Camilla Poesio, Andrea Ciampani e Michela Ponzani (gli ultimi due saggi in tedesco), presenti in: QFIAB 92 (2012).

43 Massimo Montanari, Ricordo di Vito Fumagalli (1938-1997), in: Ideologie e pratiche del reimpiego nell'alto Medioevo, 16-21 aprile 1998, Spoleto 1999 (Settimane di studio del Centro italiano di studi sull'alto medioevo 46), pp. 1-24, alle pp. 5 e 20 con nota 46.

44 Vito Fumagalli, Le origini di una grande dinastia feudale. Adalberto-Atto di Canossa, Tübingen 1971 (Bibliothek des Deutschen Historischen Instituts in Rom 35). Riferimenti a Karl Schmid in particolare a p. 52. 
Nel ruolo di assistente subentrò a Fumagalli Livia Fasola, che pose mano ad altrettanti lavori di indirizzo prosopografico; ma dopo di lei il posto di assistente non venne più ricoperto: „la situazione attuale delle università italiane non permette di prevedere quando il posto di assistente ospite italiano potrà essere occupato“, osservava Elze nel $1974 .{ }^{45}$ Attratti dal prestigio dell'Istituto si avvalsero comunque di altre sue borse Pietro Rossi, Giuseppe Frasso, Mariarosa Cortesi; ${ }^{46}$ e poi Michele Ansani, Roberto Delle Donne, Maria Pia Alberzoni, Andrea Zorzi, fino alle ultime generazioni di Barbara Bombi, Sara Menzinger e altri, che si ritrovano fra gli autori dei saggi e delle importanti recensioni. Le borse al DHI continuano ad essere tuttora ricercate non solo per il loro pregio, ma anche per la cronica mancanza di finanziamenti per postdocs nelle università italiane; il DHI, come altre istituzioni tedesche, si trova a svolgere una funzione sussidiaria per i giovani medievisti italiani, preparandone in alcuni casi la ,fuga' in Germania.

Nonostante il flusso di giovani borsisti (sette fra 1966 e 1988), fino al nuovo millennio non si può dire che in Italia vi sia stata una effettiva familiarità con la rivista. La lingua ne è stato l'ostacolo principale, insieme con l'inclinazione dei medievisti della Penisola, almeno fino agli anni Novanta, a volgersi piuttosto verso la storiografia francese e in parte inglese. Fu probabilmente la volontà di far conoscere a un più vasto pubblico italiano i risultati della ricerca tedesca a spingere Hartmut Hoffmann nel 1971 a recensire in italiano due volumi di Kienast e, tre anni dopo, Hermann Jakobs a presentare in un saggio „Sul sorgere di una nobiltà urbana“ il corposo volume su Lucca altomedievale di Hansmartin Schwarzmaier. ${ }^{47}$ Quasi un privilegium amoris si può cogliere nel piccolo gruppo di studiosi raccolti attorno a Cinzio Violante e Ottorino Bertolini che negli anni Sessanta, insieme con il direttore del DHI, Gerd Tellenbach, presero l'iniziativa di organizzare dei colloqui binazionali. Al primo, svoltosi nel 1965 a Roma su invito del DHI e su questioni riguardanti la storia sociale e locale italiana, ${ }^{48}$ ne seguirono diversi con cadenza biennale e in alternanza fra Roma e Pisa. ${ }^{49}$ Del

45 Jahresbericht 1973, in: QFIAB 54 (1974), pp. VII-XX, alle pp. IX sg.

46 Secondo il Verzeichnis der Mitarbeiter des Deutschen Historischen Instituts in Rom seit 1888, in: Deutsches Historisches Institut (vedi nota 1), pp. 99-111, in particolare pp. 105-111: V. Fumagalli 1966; L. Fasola 1970-1073; P. Rossi 1975, 1977-1978; F. Graiff 1975-1976, L. Bianconi 1976-1977; G. Frasso 1977; M. Cortesi 1978-1980.

47 Hartmut Hoffmann, Gens e duca. Due importanti nuove pubblicazioni, in: QFIAB 51 (1971), pp. 585-590. I due volumi recensiti erano: Walther Kienast, Studien über die französischen Volksstämme des Frühmittelalters, Stuttgart 1968, e id., Der Herzogstitel in Frankreich und Deutschland (9. bis 12. Jahrhundert), München-Wien 1968; Hermann Jakobs, Sul sorgere d'una nobiltà urbana. Presentazione di un nuovo libro, in: QFIAB 54 (1974), pp. 471-482. Il volume recensito, „nato nell'ambito degli studi toscani dell'Istituto Storico Germanico in Roma, sotto l'egida di Gerd Tellenbach“ (p. 471), era: Hansmartin Schwarzmaier, Lucca und das Reich bis zum Ende des 11. Jahrhunderts. Studien zur Sozialstruktur einer Herzogsstadt in der Toskana, Tübingen 1972 (Bibliothek des Deutschen Historischen Instituts in Rom 41).

48 Cfr. Jahresbericht 1965, in: QFIAB 46 (1966), p. XII; cfr. Esch, Forschungen (vedi nota 17), p. 205. 49 Esch, Forschungen (vedi nota 17), pp. 205 sg. 
secondo, tenutosi nel marzo 1968 su invito di Violante a Pisa, riferì lo „Jahresbericht“ nel fascicolo del $1969,{ }^{50}$ fascicolo in cui trovarono spazio anche due delle relazioni (Fumagalli e Keller) che erano state presentate e discusse appunto a Pisa insieme con quelle di Kurze, Schwarzmaier, Picasso, Cammarosano e Fasola. Del terzo e quarto incontro parlarono gli „Jahresberichte“ del 1970 e del 1972.51

Il legame con la scuola pisana e milanese scaturiva, oltre che da affinità tematiche e metodologiche, dal comune ambito d'indagine, quelle Toskanaforschungen che erano state uno degli Schwerpunkte dell'Istituto rivitalizzati da Tellenbach. ${ }^{52} \mathrm{E}$ questo il campo in cui credo che le QFIAB abbiano inciso più durevolmente sulla medievistica italiana e internazionale. Gli studiosi impegnati nelle ricerche toscane pubblicarono regolarmente i loro risultati nella rivista: Hansmartin Schwarzmaier, Hubert Mordek, Hagen Keller e Thomas Szabó, del quale ultimo Mauro Ronzani nel 1992 avrebbe tradotto in italiano il saggio su „Strade e sicurezza“ apparso nelle QFIAB del $1977,{ }^{53}$ contribuendo alla sua ampia ricezione. E con essi Wilhelm Kurze, autore dal 1964 di vari saggi sui monasteri toscani e curatore di uno dei progetti più longevi del DHI: il „Codice diplomatico di S. Salvatore del Monte Amiata“, concluso alla sua morte da uno dei borsisti italiani dell'Istituto, Mario Marrocchi. ${ }^{54}$ Dalla collaborazione di quei ricercatori con il gruppo di Violante e Bertolini scaturì il convegno spoletino su „Lucca e la Tuscia nell'alto medioevo“ $(1971)^{55}$ e la serie dei convegni sui ceti dirigenti in Toscana a partire dal 1978, insieme con altre iniziative. ${ }^{56}$

Il gruppo pisano-milanese appare dunque il principale motore delle relazioni con il DHI nel secondo dopoguerra. La scuola torinese di Giovanni Tabacco, che pure fu meritorio divulgatore della storiografia tedesca nella Penisola, non ha invece una particolare presenza nelle QFIAB se non tramite un unico saggio di Renato Bordone, che

50 Jahresbericht 1968, in: QFIAB 49 (1969), pp. VII-XV, alle pp. XIII sg.

51 Jahresbericht 1970, in: QFIAB 51 (1971), pp. VII-XVI, a p. XV; Jahresbericht, in: QFIAB 53 (1973), pp. VII-XVI, a p. XIII.

52 Elze, I cento anni (vedi nota 2), p. 80, e soprattutto Esch, Forschungen (vedi nota 17), pp. 203206., ibid., pp. 194-203, sulle prime Toskanaforschungen e su Fedor Schneider, che dal 1905 pubblicò numerosi saggi nelle QFIAB.

53 Thomas Szabó, Straßenbau und Straßensicherheit im Territorium von Pistoia (12.-14. Jh.). Untersuchungen zur Verkehrspolitik einer mittelalterlichen Kommune, in: QFIAB 57 (1977), pp. 88-137, tradotto da Mauro Ronzani col titolo: Strade e sicurezza nel territorio di Pistoia (secoli XII-XIV). Ricerche sulla politica viaria di un comune medievale (secoli XII-XIV), in: Thomas Szabó, Comuni e politica stradale in Toscana e in Italia nel medioevo, Bologna 1992, pp. 195-234.

54 Wilhelm Kurze (a cura di), Codex diplomaticus Amiatinus. Urkundenbuch der Abtei S. Salvatore am Montamiata. Von den Anfängen bis zum Regierungsantritt Papst Innozenz III. (736-1198), Tübingen 1974-2004, voll. 1-4; il volume 3,1: Profilo storico e materiali supplementari, Tübingen 2004, è a cura di Mario Marrocchi.

55 Atti del $5^{\circ}$ Congresso internazionale di studio sull'alto medioevo. Lucca e la Tuscia nell'alto Medioevo (Lucca, 3-7 ottobre 1971), Spoleto 1973.

56 Esch, Forschungen (vedi nota 17), pp. 206 sg. 
si misura con Eduard Hlawitschka; ${ }^{57}$ e non pare orientata alla collaborazione con il DHI neppure la scuola bolognese di Ovidio Capitani, che pur colloquiava con i colleghi transalpini - non a caso l'alma mater bolognese su proposta di Capitani avrebbe concesso nel 1982 la laurea honoris causa a Horst Fuhrmann. ${ }^{58}$ Tutto ciò non esclude l'alta considerazione per la storiografia tedesca relativa a Chiesa e papato che trovava sedimentazione anche nelle QFIAB - penso a Jürgen Miethke, Peter Herde, Bernhard Schimmelpfennig - tutti vivacemente presenti, insieme con il riservato Elze, ai più importanti degli „,innumerevoli convegni“ italiani..$^{59}$ L'impresa del „Repertorium Germanicum“ e le sue copiose sedimentazioni nelle QFIAB appaiono invece meno recepite e sfruttate nelle loro potenzialità, anche se ad esse si può accostare l'edizione dei „Libri annatarum“ dalla Camera apostolica inaugurata da Michele Ansani. ${ }^{60}$ Il quale nelle QFIAB ha peraltro presentato l'originale progetto di edizione online del „Codice diplomatico della Lombardia medievale““, ${ }^{61}$ segno di un dialogo sulla ricerca di base che nella rivista trovava collocazione ideale.

Soprattutto sotto la direzione di Arnold Esch e Michael Matheus la rete dei rapporti italiani si è via via allargata al di fuori delle classiche scuole dell'avanzato dopoguerra - ormai dissolte - e della cerchia romana, sulla quale si ritornerà. Dagli anni Novanta si è difatti rafforzato il rapporto con Cosimo Damiano Fonseca e Hubert Houben per le ricerche sui castelli di epoca Staufer nell'Italia meridionale. La tematica risale alla lungimiranza di Kehr, che nel 1905 aveva creato presso l'Istituto una sezione di storia dell'arte diretta da Arthur Haseloff ${ }^{62}$ e indirizzato quest'ultimo al censimento e allo studio dei monumenti dell'epoca Staufer, ${ }^{63}$ con un'impostazione interdisciplinare ante litteram. ${ }^{64} \mathrm{Da}$ allora il progetto di ricerca ha conosciuto diverse e non volute battute d'arresto. Il principale collaboratore di Haseloff, Eduard Sthamer, pubblicò nelle QFIAB quattro saggi, di cui solo uno su un castello; ${ }^{65}$ la sua morte

57 Renato Bordone, Un'attiva minoranza etnica nell'alto medioevo. Gli Alamanni del comitato di Asti, in: QFIAB 54 (1974), pp. 1-57.

58 Cfr. URL: https://archiviostorico.unibo.it/it/patrimonio-documentario/lauree-honoris-causa? record=131217; 20.9.2020.

59 L'espressione, carica di una certa qual critica rassegnazione, è ripetuta dal direttore Esch nel 1990 e nel 1991: Jahresbericht 1989, in: QFIAB 70 (1990), pp. VII-XXIII, a p. VII; Jahresbericht 1990, in: QFIAB 71 (1991), pp. IX-XXVIII, a p. XVIII.

60 Michele Ansani (a cura di), Camera apostolica. Documenti relativi alle diocesi del Ducato di Milano (1458-1471), vol. 1: I „libri annatarum“ di Pio II e Paolo II, Milano 1994.

61 URL: http://www.lombardiabeniculturali.it/cdlm/; 20.9.2020; sull'iniziativa cfr. Michele Ansani/Valeria Leoni, Experiment einer digitalen Edition urkundlicher Quellen. Der Codice diplomatico della Lombardia medievale (8.-12. Jahrhundert), in: QFIAB 86 (2006), pp. 538-561.

62 Elze, I cento anni (vedi nota 2), p. 71.

63 Houben, Hundert Jahre (vedi nota 36), p. 108.

64 Così Arnold Esch: Arnold Esch/Andreas Kiesewetter, Süditalien unter den ersten Angiovinen. Abschriften aus den verlorenen Anjou-Registern im Nachlaß Eduard Sthamer, in: QFIAB 74 (1994), pp. 646-663, a p. 647.

65 Eduard Sthamer, Zur Geschichte des Kastells Rocca S. Agata, in: QFIAB 15 (1913), pp. 390-396. 
prematura, oltre ad altre vicende, impedì di valorizzare le sue sterminate ricerche sui registri angioini. ${ }^{66}$ Quando però nel 1994 Arnold Esch e Andreas Kiesewetter pubblicarono nelle QFIAB la notizia e l'inventario del suo lascito, che era stato individuato l'anno prima da Reinhard Elze fra i materiali giunti da Berlino alla sede dei Monumenta Germaniae Historica (MGH) a Monaco dopo la Wende, si avviarono sia nuove indagini impostate su base archeologica sia studi come quelli di Kristjan Toomaspoeg, che sfruttavano i regesti Sthamer per prendere in esame altre problematiche. ${ }^{67}$ L'Italia meridionale, che già con Norbert Kamp, Dieter Girgensohn, Vera von Falkenhausen e altri aveva suscitato l'interesse dei collaboratori del DHI specie per l'età normanno-sveva, è sicuramente una delle frontiere attuali della ricerca internazionale che fa capo al DHI e alle QFIAB, ${ }^{68}$ anche grazie alle indagini interdisciplinari per il Mezzogiorno svevo-angioino (storia e archeologia) promosse da Michael Matheus durante la sua direzione. Il progetto di Kordula Wolf e Marco Di Branco su religioni, culture e poteri politici fra età longobarda e normanna, la trascrizione ed edizione dei documenti di Ruggero I di Sicilia, le ricerche su cristiani e musulmani nella Capitanata del Duecento ${ }^{69}$ sono stati di recente inseriti in un più ampio quadro di storia del Mediterraneo, che ambisce a una dimensione transculturale e transepocale. ${ }^{70}$

Una traccia visibile hanno lasciato nelle QFIAB le special relations dell'Istituto con l'ambiente romano. Ancora una volta decisivo è stato l'impulso dato da Paul F. Kehr all'edizione dei „Regesta chartarum“, pubblicati insieme con l'Istituto Storico Italiano per il Medioevo ${ }^{71}$ e con un logo che raffigurava insieme „i due grandi rappresentanti della ricerca storica italiana e tedesca, Muratori e Leibniz, il cui tentativo di una comune ricerca è stato ripreso dai due istituti “ ${ }^{72} \mathrm{Nel}$ secondo dopoguerra la collaborazione con l’Istituto Storico Italiano per il Medioevo (ISIME) è stata rinnovata

$66 \mathrm{Su}$ tali vicende ampiamente Houben, Hundert Jahre (come nota 36), pp. 112-126.

67 Cfr. Kristjan Toomaspoeg, Decimae. Il sostegno economico dei sovrani alla Chiesa del Mezzogiorno nel XIII secolo. Dai lasciti di Eduard Sthamer e Norbert Kamp, Roma 2009 (Ricerche dell'Istituto Storico Germanico di Roma 4), Roma 2009.

68 Ancora Houben, Hundert Jahre (vedi nota 36), pp. 124-129.

69 Cfr. rispettivamente la bibliografia indicata sul sito del progetto „Fluider Grenzraum. Das frühmittelalterliche Unteritalien im Spannungsfeld rivalisierender Religionen und politischer Mächte (9. - Anfang 10. Jahrhundert)“ (URL: http://www.dhi-roma.it/aktuelle-projekte.html; 20.9.2020); Julia Becker (a cura di), Documenti latini e greci del conte Ruggero I di Calabria e Sicilia. Edizione critica, Roma 2013 (Ricerche dell'Istituto Storico Germanico di Roma 9); Jahresbericht 2009, in: QFIAB 90 (2010), pp. IX-LXI, a p. XXIX. Tra le ricerche relative all'ultimo progetto citato vanno ricordati gli importanti scavi avviati da Lukas Clemens e Michael Matheus. I risultati sono pubblicati in: Lukas Clemens/Michael Matheus (a cura di), Christen und Muslime in der Capitanata im 13. Jahrhundert: Archäologie und Geschichte, Trier 2018.

70 Jahresbericht 2015, in: QFIAB 96 (2016), pp. IX-LXX, a p. IX.

71 Elze, I cento anni (vedi nota 2), p. 70; Esch, Forschungen (vedi nota 17), pp. 193-198.

72 Kehr ne parla nello Jahresbericht del 1903/04, in: QFIAB 7 (1904), p. 6. La citazione dallo Jahresbericht des Historischen Instituts 1906-07, in: QFIAB 10 (1907), pp. I-X, a p. VI. 
e approfondita, si pensi al „nuovo Potthast““ ${ }^{73}$ ma anche alla duplice attività di Wolfgang Hagemann: impegnato nella ricerca e nella pubblicazione sulle QFIAB di „Kaiserurkunden und Reichssachen“, alle quali era stato avviato quando aveva iniziato la sua attività all'Istituto nel 1935, ${ }^{74}$ Hagemann contemporaneamente proseguì le sue ricerche per l'edizione dei diplomi di Ludovico II „per l’ISIME“ (1969), edizione poi portata a compimento da Konrad Wanner. ${ }^{75}$

È di pochi anni dopo (1974) la nascita del Circolo Medievistico Romano ad opera di Reinhard Elze, insieme con Girolamo Arnaldi (ISIME) e André Vauchez (École française de Rome). I seminari interdisciplinari organizzati annualmente dal Circolo „sono diventati nel corso degli anni un importante luogo di scambi e incontri scientifici tra diversi paesi e diverse discipline della medievistica (storici, archeologi, storici dell'arte, filologi, filosofi.... “; ${ }^{76}$ regolarmente segnalati nelle QFIAB, dal 1993 sono valorizzati tramite una specifica voce dell'indice generale e una breve sintesi dei contenuti. ${ }^{77}$ Ad Andreas Rehberg, referente del DHI per il Circolo, si deve la cura dello Schwerpunkt sulla storia della città di Roma, introdotto fra i progetti dell'Istituto grazie alla tenacia del direttore Arnold Esch; $;^{78}$ con una serie di ricerche sullo Studium, sull'araldica, sulle sepolture femminili fra Medioevo e Rinascimento, Rehberg indaga la storia civile romana non solo nelle $\mathrm{QFIAB},{ }^{79}$ ma pure in riviste gemelle come $\mathrm{i}$ „Mélanges de l’École française de Rome - Moyen Âge““, 80 segno anche questo della fertile collaborazione con colleghi italiani e stranieri.

Il Circolo rappresenta una delle più importanti iniziative di coordinamento fra gli innumerevoli istituti di ricerca stranieri con sede a Roma, che „è infatti, grazie ai suoi istituti scientifici italiani e stranieri, un centro di ricerca internazionale unico al

73 URL: https://www.isime.it/public/archivio/materiali-pdf/Inventario_Repertorio.pdf; 20.9.2020.

74 Elze, I cento anni (vedi nota 2), p. 74.

75 Jahresbericht 1968, in: QFIAB 49 (1969), pp. VII-XV, a p. X. Alla morte di Hagemann nel 1978 il lavoro fu ripreso da Konrad Wanner, cfr. Konrad Wanner, Vorrede, in: Ludovici II Diplomata / Die Urkunden Ludwigs II., Roma 1994 (Fonti per la Storia dell'Italia medievale. Antiquitates 4) = MGH DD Lu II, pp. VII-VIII, a p. VIII.

76 Cito dalla presentazione del sito, URL: https://circolomed.hypotheses.org/; 20.9.2020.

77 Vorträge des Circolo Medievistico Romano 1992/1993, in: QFIAB 73 (1993), pp. 696 sg.

78 In una nota dello Jahresbericht del 1999, A. Esch esprime la soddisfazione per l'istituzione del ruolo di ricercatore dedicato alle Fonti per la storia della città di Roma nel basso Medioevo e nel Rinascimento, ruolo previsto nel 1988 e solo dieci anni dopo realizzato, cfr. Jahresbericht 1999, in: QFIAB 80 (2000), pp. VII-XXXIII, a p. XX.

79 Ad esempio Martin Bertram/Andreas Rehberg, Matheus Angeli Johannis Cinthii. Un commentatore romano delle Clementine e lo Studium Urbis nel 1320, in: QFIAB 77 (1997), pp. 84-143; Andreas Rehberg, Die ältesten erhaltenen Stadtratsprotokolle Roms (1515-1526). Teil III. Kommentar und Indizes, in: QFIAB 82 (2002), pp. 231-403.

$80 \mathrm{Si}$ veda ad esempio Andreas Rehberg, Aspetti araldici delle sepolture femminili romane del Rinascimento, in: Donne di Pietra. Immagini, vicende, protagoniste delle sepolture romane del Rinascimento: una ricerca in corso, in: Mélanges de l'École française de Rome. Moyen Âge 127 (2015), pp. $35-46$. 
mondo“. ${ }^{81}$ Qui la rivista ha trovato opportunità per sviluppare collaborazioni e reti, con un'intensità sorprendente nel corso degli ultimi decenni. Accanto a un interlocutore in qualche modo predestinato e privilegiato quale l'ISIME, risaltano i legami intrecciati con la Bibliotheca Hertziana, l’Österreichisches Institut, l'École française, l'„Enciclopedia Italiana di scienze, lettere ed arti“ e altri centri di ricerca, in occasione di convegni, mostre, workshops. Ne riferiscono gli „Jahresberichte“ e poi le cronache dettagliate all'interno della rivista, che così ne accelerano e intensificano la ricezione. Va poi ricordata la nascita nel 2007, presso la casa editrice romana Viella, delle „Ricerche dell'Istituto Storico Germanico di Roma“, una nuova collana in lingua italiana che intende „far conoscere le ricerche dell'istituto a un più ampio pubblico italiano interessato alla ricerca“" ${ }^{82}$ Le monografie di argomento medievistico finora apparse nella collana sono tutte opera di borsisti dell'Istituto, già presenti con i loro temi di ricerca nelle QFIAB. ${ }^{83}$

La rivista si avvantaggia insomma del circolo virtuoso instaurato fra iniziative dell'Istituto e loro pubblicazione, nonché della capacità di direttori e collaboratori d'entrare in dialogo proficuo con università sia tedesche - Mainz, Heidelberg, Monaco - sia italiane - la Sapienza, Roma Tre, Genova; e, ancora, con l'Istituto storico italo-germanico, lo European University Institute (EUI) di Firenze, I Tatti. L'internazionalizzazione della ricerca ha trovato nell'Istituto e nella sua rivista un ambiente di coltura ideale: partendo dalle intuizioni e dalle capacità organizzative dei suoi direttori e collaboratori, sfruttando la sua base binazionale e il mondo cosmopolita della capitale romana.

Una sezione della rivista che si è progressivamente ampliata è quella bibliografica, dotata di recensioni spesso anche in lingua italiana. Fin dai primi anni vi s'intendeva rendere conto della letteratura italiana e straniera su Italia e papato, nonché sul loro rapporto con gli altri paesi, dal Cinquecento ad oggi. ${ }^{84}$ Nei primi 17 volumi il secondo segretario dell'Istituto prussiano, Karl Schellhass, s’impegnò a „raccogliere le novità editoriali più importanti nel campo della storia italiana medievale e moderna (libri e saggi), allo scopo di farle conoscere ai lettori tedeschi della rivista“, per un totale di circa 1200 pagine e più di 26.000 titoli. ${ }^{85}$ Impressiona per sistematicità e

81 Elze, I cento anni (vedi nota 2), p. 56.

82 Jahresbericht 2007, in: QFIAB 88 (2008), pp. IX-LVI, a p. X. Come recita il sito di Viella, „nella collana in lingua italiana si pubblicano edizioni critiche, nonché monografie e volumi collettanei di carattere scientifico relativi alla storia italiana e italo-tedesca dal primo medioevo al passato più recente“ (URL: https://www.viella.it/catalogo/collana/26; 20.9.2020).

83 Sono le monografie di Barbara Bombi, Kristjan Toomaspoeg, Sara Menzinger (insieme con Emanuele Conte), Julia Becker. Alle monografie si aggiungono gli atti del convegno Rehberg/Esposito, cfr. URL: https://www.viella.it/libro/9788883342615; 20.9.2020.

84 Così Schellhass nel 1913: Bibliographie zusammengestellt von Karl Schellhass, in: QFIAB 15 (1913), pp. 159-221, 400-440, a p. 400 (Vorbemerkung).

85 Elze, I cento anni (vedi nota 2), p. 72. 
chiarezza di disegno la suddivisione di Schellhass, che distingue e valorizza scritti sul metodo storico, progetti di lavoro, sommari di archivi e cataloghi di biblioteche, per poi categorizzare e comprendere tutte le discipline storiche, dalle cosiddette scienze ausiliarie alla storia sociale, alla geografia e agiografia, alla teologia, all'etnografia. ${ }^{86}$ Insieme con il „Deutsches Archiv für Erforschung des Mittelalters“ (DA), con gli „Studi medievali“ e prima del „Medioevo Latino“(MEL), le recensioni delle QFIAB sono state uno degli strumenti di aggiornamento più proficuo per gli studi medievali - in particolare per i medievisti italiani privi di grande confidenza con la lingua tedesca. Tuttora, grazie alla pubblicazione online, le QFIAB assolvono tale compito egregiamente.

Nel 2017 Martin Baumeister osservava che iniziative e progetti dell'Istituto emergono „mit einer dezidiert transnationalen bzw. transregionalen und zugleich interdisziplinären Ausrichtung ..., die oft auch globalgeschichtliche Perspektiven und Problemstellungen mit einbezogen“. E sottolineava, ,wie sehr sich das Institut ... immer mehr über die weiterhin grundlegende binationale Orientierung hinaus als Knotenpunkt und Laboratorium transregionaler und transnationaler Studien versteht“, tanto che nelle sue ricerche „Vormoderne und Moderne eng miteinander verknüpft und in Beziehung zueinander gesetzt werden“. ${ }^{87}$ In queste poche righe si delinea come un manifesto della ricerca storica contemporanea, che l'Istituto ha fatto proprio e proietta anche sulle QFIAB. In tale prospettiva, la rivista si colloca all'avanguardia degli studi e si apre a un futuro promettente. Da medievisti si può solo desiderare che essa mantenga la cura amorosa per un Medioevo che negli ultimi decenni tende a stemperarsi in una indistinta Vormoderne, a sua volta inghiottita, a partire dalle università, dalla Moderne. Saltati i confini spazio-temporali ed epistemologici della disciplina tradizionale, ristretti ma rassicuranti, la medievistica naviga in mare aperto. È una sfida che certo vale la pena di affrontare, ma senza sacrificare il grande patrimonio filologico che l'Otto e Novecento ci hanno consegnato. In questo senso credo che le QFIAB debbano preservare quella vocazione originaria alla ricerca di base negli archivi vaticani e italiani che rende la rivista e il suo Istituto tuttora inconfondibile nell'affollato panorama scientifico internazionale. Per far sì che nella sua Profilbildung permanga l'eredità del „Repertorium Germanicum“, da tutelare quanto a fonti di finanziamento e arco cronologico, magari da rilanciare, mettendo da parte il ,germanico“ per puntare al „transnazionale“, recuperando quella sperimentalità ardita nel pensare e rimodellare le fonti che quel progetto presentava.

Quellen und Forschungen, una coppia felice che celebra nozze centenarie. Ad multos annos!

86 Cfr. Bibliographie zusammengestellt von Karl Schellhass, in: QFIAB 10 (1907), pp. 379-469, a p. 379; Bibliographie zusammengestellt von Karl Schellhass, in: QFIAB 11 (1908), pp. 371-475, a p. 371. 87 Jahresbericht 2016, in: QFIAB 97 (2017), pp. IX-LXX, alle pp. IX sg. 